\title{
Ultrahigh-Energy Photons as Probes of Lorentz Symmetry Violations in Stringy Space-Time Foam Models
}

\author{
Luca Maccione, ${ }^{1, *}$ Stefano Liberati, ${ }^{2,3, \dagger}$ and Günter Sigl ${ }^{4, \$}$ \\ ${ }^{1}$ DESY, Theory Group, Notkestraße 85, D-22607 Hamburg, Germany \\ ${ }^{2}$ SISSA, Via Bonomea 265, 34136 Trieste, Italy \\ ${ }^{3}$ INFN, Sezione di Trieste, Via Valerio, 2, I-34127, Trieste, Italy \\ ${ }^{4}$ II. Institut für Theoretische Physik, Universität Hamburg, Luruper Chaussee 149, D-22761 Hamburg, Germany \\ (Received 9 April 2010; revised manuscript received 4 June 2010; published 9 July 2010)
}

\begin{abstract}
The time delays between $\gamma$ rays of different energies from extragalactic sources have often been used to probe quantum gravity models in which Lorentz symmetry is violated. It has been claimed that these time delays can be explained by or at least put the strongest available constraints on quantum gravity scenarios that cannot be cast within an effective field theory framework, such as the space-time foam, $D$-brane model. Here we show that this model would predict too many photons in the ultrahigh energy cosmic ray flux to be consistent with observations. The resulting constraints on the space-time foam model are much stronger than limits from time delays and allow for Lorentz violation effects way too small for explaining the observed time delays.
\end{abstract}

DOI: 10.1103/PhysRevLett.105.021101

Introduction.-Recent years have witnessed a growing interest in possible small deviations from the exact local Lorentz invariance (LI) of general relativity. On the theoretical side, ideas stemming from the quantum gravity (QG) community led to conjecture that LI may not be an exact local symmetry of the vacuum. On the observational side, high energy astrophysics observations played a leading role in constraining such models and, in particular, the recent detection of time delays on arrival of high energy $\gamma$ rays $[1,2]$ led to renewed interest of the astrophysics community in QG induced Lorentz violation (LV) effects. For a comprehensive review see, e.g., [3-6].

The observed time delays can be explained, and are actually expected, in standard astrophysical scenarios, hence, they can be readily used to place constraints on LV models. However, time delays are naturally predicted also in generic LV QG models. Hence, the possibility that QG effects could account for all or part of the observed phenomenology raised a vibrant interest.

It is now established that any LV model able to reproduce the observed delays and admitting an effective field theory (EFT) formulation is in tension with other astrophysical observations (see, e.g., [6]). Up to now, the only fully developed LV model able to explain the observed time delays has a string theory origin and does not admit an EFT formulation [7-14]. We shall not consider here the possible alternative of deformed special relativity models $[5,15]$ as their dynamics is not fully developed to date. In particular, in [7] it is not only suggested that the considered QG model could possibly account for all the observed time delays, but it is shown that it might also explain consistently the dark energy content of the Universe.

Therefore, if observed time delays were due to such QG effects, the propagation of $\mathrm{GeV}$ photons over cosmological
PACS numbers: $98.70 . S a, 04.60 .-\mathrm{m}, 11.30 . \mathrm{Cp}, 96.50 . \mathrm{sb}$

distances could not be described within EFT. Given that EFT is accurately verified with terrestrial accelerators up to $\sim 100 \mathrm{GeV}$, this would be a very striking and revolutionary conclusion that calls for extra investigations of stringy QG models.

In this Letter we show that experimental data on the photon content of ultrahigh-energy cosmic rays (UHECRs) lead, for the first time, to strong constraints on this $D$-brane LV model, making it unsuitable to consistently explain the observed time delays and probably unnatural from a theoretical point of view. As we will argue in the following, due to suppression of ultrahigh-energy (UHE) photon absorption on intergalactic radiation fields, the fraction of photons in UHECRs predicted within this model would be so large as to violate present experimental limits.

Time delays.-Effects suppressed by the Planck scale $M_{\mathrm{Pl}}=1.22 \times 10^{19} \mathrm{GeV}$ are in principle hard to detect. Yet in some peculiar situations these tiny effects can be possibly magnified and become sizable. In order to identify these situations, it is required to work in a well-defined theoretical framework to describe particle dynamics.

In the model [7-14] only purely neutral particles, such as photons or Majorana neutrinos, possess LV modified dispersion relations. For photons this has the form

$$
E_{\gamma}^{2}=p^{2}-\xi \frac{p^{\alpha}}{M^{\alpha-2}},
$$

with the free parameter $\xi>0$. Hence, only subluminal photons are present in the theory, and photon propagation in vacuum is not birefringent. In particular, the model outlined in [7-14] predicts $\alpha=3$, hence we will fix $\alpha=$ 3 in the following. Because of stochastic losses in interactions with the $D$-brane foam, exact energy-momentum conservation during interactions does not hold. This last 
phenomenon is controlled by the free parameter $\xi_{I}$ [8], in a way which we will clarify below. Because both $\xi$ and $\xi_{I}$ are dimensionless, their natural values are $\mathcal{O}(1)$, and constraints stronger than $\mathcal{O}(1)$ mean that extra suppression of the LV effects has to be invoked.

This model evades most of the present constraints. The electron and birefringence constraints discussed in [6] do not apply, because the theory has LV only in the photon (and Majorana neutrino) sector, it is not birefringent, and LV applies only to real (on shell) particles [14]. UHECR constraints [16-21] do not apply as well.

However, according to Eq. (1) photons with different energy travel at different speeds. Then, if a source at redshift $\bar{z}$ simultaneously emitted two photons at energy $E_{1}^{\prime} \neq E_{2}^{\prime}$, their time delay at Earth will be

$$
\Delta t \simeq \xi \frac{\Delta E}{M} \frac{1}{H_{0}} \int_{0}^{\bar{z}} d z \frac{1+z}{\sqrt{\Omega_{\Lambda}+(1+z)^{3} \Omega_{M}}},
$$

where $\Delta E$ is the observed energy difference and the integral on redshift accounts also for redshift of the energy [22-24]. Time-of-flight constraints are then viable for this model, even though they lead at most to constraints on $\xi$, because $\xi_{I}$ is not effective in this context.

Rather intriguingly, the FERMI Collaboration has recently reported the detection of delays on arrival of $\gamma$-ray photons emitted by distant gamma-ray bursts (GRBs), in particular, GRB 080916C [1] and GRB 090510 [2] (see, however, [23] for an updated review). A thorough analysis of these delays in the energy range $35 \mathrm{MeV}-31 \mathrm{GeV}$ allowed us to place for the first time a conservative constraint of order $\xi \lesssim 0.8$ [2] on LV effects expressed as in Eq. (1). This is the best constraint so far available on the theory. On the other hand, FERMI results can be interpreted in terms of $\mathrm{LV}$ assuming $\xi \simeq 0.4$ and a possible evolution of the $D$-particle density with redshift [7]. Plausible astrophysical explanations of this phenomenon exist. No claim of a discovery of LV can be made on the basis of the data reported in $[1,2]$, where only LV constraints are discussed.

Photon absorption in D-brane models. - In order to constrain the $D$-brane model, we exploit the process of pair production, $\gamma \gamma \rightarrow e^{+} e^{-}$, which is, in particular, responsible for the absorption of UHE photons produced in Greisen-Zatsepin-Kuzmin (GZK) interactions [25,26]. Indeed, if GZK energy losses affect the propagation of UHECR protons in the intergalactic medium, then a large amount of UHE photons is generated by the decay of the $\pi^{0}$ 's copiously produced in such interactions. UHE photons are attenuated by pair production onto the cosmic microwave background (CMB) and radio background during their travel to Earth, leading to their fraction in the total UHECR flux being reduced to less than $1 \%$ at $10^{19} \mathrm{eV}$ and less than $10 \%$ at $10^{20} \mathrm{eV}[27,28]$. It was shown in a framework with modified dispersion relations for both photons and $e^{+}, e^{-}$and standard energy-momentum conservation, that pair production could be effectively inhibited at high energy, due to the presence of an upper threshold [29], and therefore the fraction of photons present in UHECRs on Earth would violate the present experimental upper limits. An upper threshold is an energy above which it is not possible to simultaneously conserve energy and momentum in an interaction. If Lorentz symmetry is exact then upper thresholds do not exist, while they might well exist if it is violated [30]. Hence, the nonobservation of a large fraction of UHE photons in UHECRs implies the constraint $|\xi|<\mathcal{O}\left(10^{-14}\right)$ in the EFT framework $[31,32]$.

We want to address here the problem of whether the same argument can be applied in the space-time foam model with energy nonconservation. First, we note that $\gamma$ rays are indeed generated by $\pi^{0}$ decay: In $D$-brane models LV acts as an energy dependent modification of the background space-time metric (the new metric is of Finslerian type $[14,33])$, hence, it can act only on real particles. But in order for $\pi^{0}$ 's to decay, they must excite modes of the electromagnetic vacuum, i.e., virtual photons, which then are not affected by LV. The LV effects described in $D$-brane models are the result of multiple interactions between photons and $D$ branes, hence, cannot be relevant in the mere process of photon production. In fact, this seems to justify a more general theorem, stating that only reactions with photons in the initial state can be affected by LV in this model. Note that the resulting violation of time reversal invariance is due to LV in this scenario. Moreover, $\pi^{0}$ is not a structureless particle, and its constituents are charged, hence, they do not interact with $D$ particles. Hence, $\pi^{0}$ decay is not affected by LV.

Now we consider the threshold equations [8]

$$
E_{1}+\omega=E_{2}+E_{3}+\delta E_{D} \quad p_{1}-\omega=p_{2}+p_{3},
$$

where $\omega$ is the energy of the low energy background photon ( $\omega \simeq 6 \times 10^{-4} \mathrm{eV}$ for a CMB photon), $E_{1} \simeq p_{1}-$ $(\xi / M) p_{1}^{2} / 2$ is the energy of the high energy photon and $E_{j} \simeq p_{j}+m_{e}^{2} /\left(2 p_{j}\right)$, with $j=2,3$ are the energies of the outgoing electron and positron. The symbol $\delta E_{D}$ represents the energy lost in the stochastic interactions with the $D$ branes. The above equation is already written in the threshold configuration [30]. We exploit momentum conservation and the ultrarelativistic limit, to get

$$
2 \omega-\frac{m_{e}^{2}}{2 p_{2}}-\frac{m_{e}^{2}}{2 p_{3}}=\delta E_{D}^{(4)}+\frac{\xi}{M}\left(\omega^{2}+\omega\left(p_{2}+p_{3}\right)+p_{2} p_{3}\right)
$$

where $\delta E_{D}^{(4)}$ is the amount of energy violation in a fourparticle interaction, and corresponds to the sum of the corresponding violations in each of the two three-body interactions described by Eq. (27) in [8]

$$
\delta E_{D}^{(4)} \equiv \delta E_{D}+\frac{\xi}{2 M}\left(p_{2}^{2}+p_{3}^{2}-\omega^{2}\right) \simeq \frac{\xi_{I}}{2 M} E_{\mathrm{th}}^{2},
$$

where according to [8] we assume the last equality to hold, 
with $\xi_{I}$ different from $\xi$ in principle. While it is natural that $\delta E_{D}^{(4)}$ depends only on $E_{\mathrm{th}}$ and $M$, as they are the only energy scales of the problem, the effect of the quantum fluctuations $\delta E_{D}$ is less clear. We shall assume that this effect only amounts to a redefinition of the unknown parameter $\xi_{I}$, and check that our conclusions remain unchanged if we let $\xi_{I}$ fluctuate in the interaction up to 10 times its central value. The threshold equation can then be derived by putting $p_{2}=p_{3}=\left(p_{1}-\omega\right) / 2$ and $p_{1} \equiv E_{\mathrm{th}}$. By introducing $x \equiv E_{\text {th }} / M$ we obtain

$$
\begin{aligned}
- & \frac{\xi_{I}+\xi / 2}{2} x^{3}+\frac{\xi_{I}-\xi / 2}{2} \frac{\omega}{M} x^{2} \\
+\left(2+\frac{\xi}{4} \frac{\omega}{M}\right) \frac{\omega}{M} x-2 & \frac{\omega^{2}+m_{e}^{2}}{M^{2}}+\frac{\xi}{4}\left(\frac{\omega}{M}\right)^{3}=0 .
\end{aligned}
$$

Constraints. - If we now neglect all the terms more than linear in either $\xi$ or $\omega / M$, we recover Eq. (32) in [8]

$$
-\frac{\xi_{I}+\xi / 2}{2} x^{3}+2 \frac{\omega}{M} x-2 \frac{m_{e}^{2}}{M^{2}}+\ldots=0 .
$$

We represent (up to factors) Eq. (7) in Fig. 1, for $\xi / 2+$ $\xi_{I}=10^{-12}$, but we solve it for general values of $\xi / 2+\xi_{I}$ to establish constraints. Equation (7) has in general a lower and an upper threshold ( $E_{\text {low }}$ and $E_{\text {up }}$, respectively). From the observational requirement that $E_{\text {up }}>10^{19} \mathrm{eV}$, with $\xi_{I}$ and $\xi$ varying independently (and setting $M=M_{\mathrm{Pl}}, \omega=$ $6 \times 10^{-4} \mathrm{eV}$ and $m_{e}=0.511 \mathrm{MeV}$ ), values of $\xi_{I}, \xi>$ $10^{-12}$ are excluded by the nonobservation of a significant photon fraction in the UHECR spectrum by the Auger experiment [34].

It was recently proposed in [7] that the evolution with redshift of the $D$-particle or $D$-void background might allow us to understand why significant delays compatible with $\xi \simeq \mathcal{O}(1)$ are present in some GRBs, while in other GRBs the delays are much smaller and imply $\xi<\mathcal{O}(1)$. Since the effect of time delay (as well as the one of energy nonconservation) is expected to be proportional to the

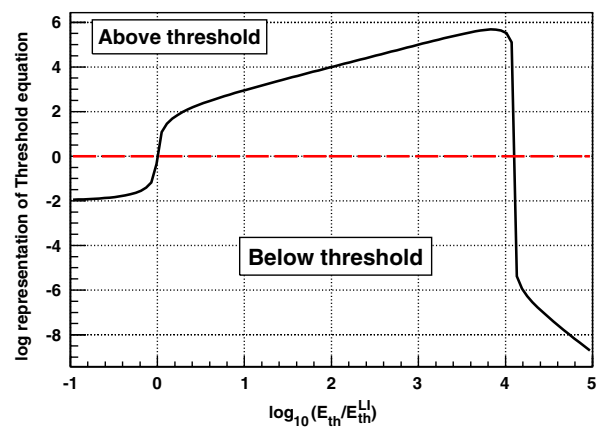

FIG. 1 (color online). Equation (7) is drawn for $\xi / 2+\xi_{I}=$ $10^{-12}$. On the $y$ axis $\operatorname{sgn}[$ Eq. (7) $] \times \log _{10}[|\mathrm{Eq} .(7)| / 0.01]$ is drawn, where 0.01 is just a small value. Positive values on the $y$ axis mean that the reaction is allowed, while negative values mean that it is forbidden. Both lower and upper thresholds are present. density of $D$ particles, the scenario envisaged by the data requires the density of $D$ particles to be large for redshift $z>1$, to drop at $z \sim 1$ and to raise again for $z<1$. This evolution might in principle affect our constraints, which depend on the effectiveness of pair production at least up to $z \sim 3$. To address this issue, we modified the public UHECR propagation code CRPropa [35].

Following [7], we assume that for $0.2<z<1 \mathrm{LV}$ effects are suppressed (then, pair production is allowed as if LI were exact and UHE photons are effectively absorbed), while outside this redshift range we assume that the LV effects are strong and that pair production absorption is inhibited (i.e., we switch it off). Because UHE photons are mainly of local origin, this assumption is conservative: Moving the suppression of LV effects to more distant epochs would indeed increase the photon fraction on Earth. We found that even in this case the photon fraction would violate experimental limits for values of $\xi, \xi_{I}>$ $10^{-12}$ (see Fig. 2).

The interactions between photons and $D$ particles might be suppressed if the momentum $\Delta p$ transferred to the $D$ particle is large compared to its mass $M_{D}=M_{s} / g_{s}$, where $M_{s}$ is the string scale and $g_{s}$ is the coupling [14]. In the standard string framework $M_{D}$ is expected to be at least of order $M_{\mathrm{Pl}}$ [7], therefore this would not be an issue for our constraint. However, in some compactification schemes, lower values of $M_{D}$ cannot be excluded [14]. If $\Delta p \gg M_{D}$, $g_{s}$ is replaced by an effective coupling $g_{s}^{\text {eff }}=g_{s} / \Gamma$, where $\Gamma \sim \Delta p / M_{D}$, and given that the unknown coefficients $\xi$ and $\xi_{I}$ are proportional to the scattering cross section, which in turn is proportional to $g_{s}^{2}$, they both receive a natural suppression $1 / \Gamma^{2}$. In order to explain the observed

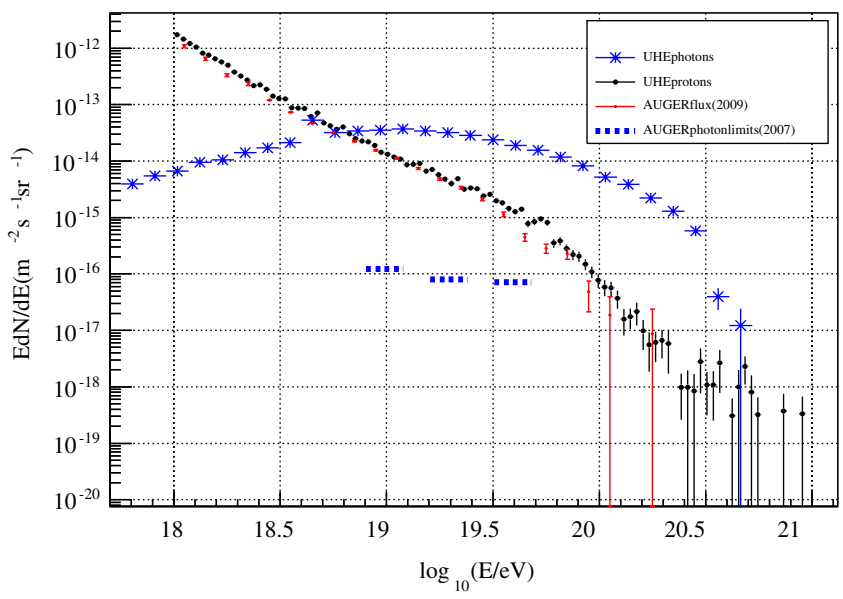

FIG. 2 (color online). UHE proton and photon simulated spectra assuming that pair production is inhibited for $z<0.2$ and $z>$ 1 for a proton injection spectrum $\propto E^{-2.5}$ up to $10^{21} \mathrm{eV}$ and source density redshift evolution as in [36]. Error bars on the simulated proton flux correspond to the statistical error of the simulation. Measured UHECR flux (in red) is from [37], while upper limits on the integral photon flux (dashed, in blue) are from [34]. The latter approximates $E d N / d E$ within a factor $\sim 3$. 
time delays in the GeV-TeV energy range within the model [14], $M_{D}$ has to be substantially larger than the TeV scale. However, on the basis of kinematics the maximum suppression factor can be estimated as being $\mathcal{O}\left(10^{10}\right)$, thereby weakening our constraint to $\xi_{I}, \xi \lesssim 10^{-2}$.

Hence, we conclude that $D$-particle explanations of GRB time delays, such as in [7], are in conflict with data on the photon fraction in UHECRs.

We thank G. Amelino-Camelia, D. Mattingly and especially N. Mavromatos for useful comments. This work was supported by the Deutsche Forschungsgemeinschaft through the collaborative research center SFB 676. L. M. and G. S. acknowledge support from the State of Hamburg, through the Collaborative Research program "Connecting Particles with the Cosmos" within the framework of the LandesExzellenzInitiative (LEXI).

Note added.-Some possible implementations of the model [12] were recently proposed [38] which would naturally evade the constraints here presented.

\section{*luca.maccione@desy.de}

†liberati@sissa.it

† guenter.sigl@desy.de

[1] A. A. Abdo et al. (Fermi LAT and Fermi GBM Collaborations), Science 323, 1688 (2009).

[2] A. A. Abdo et al., Nature (London) 462, 331 (2009).

[3] D. Mattingly, Living Rev. Relativity 8, 5 (2005), http:// www.livingreviews.org/lrr-2005-5.

[4] T. Jacobson, S. Liberati, and D. Mattingly, Ann. Phys. (N.Y.) 321, 150 (2006).

[5] G. Amelino-Camelia, arXiv:0806.0339.

[6] S. Liberati and L. Maccione, Annu. Rev. Nucl. Part. Sci. 59, 245 (2009).

[7] J. Ellis, N.E. Mavromatos, and D. V. Nanopoulos, arXiv:0912.3428.

[8] J. R. Ellis, N. E. Mavromatos, and D. V. Nanopoulos, Phys. Rev. D 63, 124025 (2001).

[9] J. R. Ellis, N. E. Mavromatos, D. V. Nanopoulos, and A. S. Sakharov, Int. J. Mod. Phys. A 19, 4413 (2004).

[10] J. R. Ellis, N.E. Mavromatos, and A.S. Sakharov, Astropart. Phys. 20, 669 (2004).

[11] J. R. Ellis, N. E. Mavromatos, and D. V. Nanopoulos, Phys. Lett. B 293, 37 (1992).

[12] J. R. Ellis, N. E. Mavromatos, and D. V. Nanopoulos, Phys. Lett. B 665, 412 (2008).
[13] J. Ellis, N. E. Mavromatos, and D. V. Nanopoulos, Phys. Lett. B 674, 83 (2009).

[14] T. Li, N. E. Mavromatos, D. V. Nanopoulos, and D. Xie, Phys. Lett. B 679, 407 (2009).

[15] G. Amelino-Camelia, Int. J. Mod. Phys. D 11, 35 (2002).

[16] L. Maccione, A. M. Taylor, D. M. Mattingly, and S. Liberati, J. Cosmol. Astropart. Phys. 04 (2009) 022.

[17] R. Aloisio, P. Blasi, P. L. Ghia, and A. F. Grillo, Phys. Rev. D 62, 053010 (2000).

[18] F. W. Stecker and S. T. Scully, Astropart. Phys. 23, 203 (2005).

[19] L. Gonzalez-Mestres, Nucl. Phys. B, Proc. Suppl. 190, 191 (2009).

[20] S. T. Scully and F. W. Stecker, Astropart. Phys. 31, 220 (2009).

[21] F. W. Stecker and S. T. Scully, New J. Phys. 11, 085003 (2009).

[22] U. Jacob and T. Piran, J. Cosmol. Astropart. Phys. 01 (2008) 031.

[23] G. Amelino-Camelia and L. Smolin, Phys. Rev. D 80, 084017 (2009).

[24] J. R. Ellis, K. Farakos, N. E. Mavromatos, V. A. Mitsou, and D. V. Nanopoulos, Astrophys. J. 535, 139 (2000).

[25] K. Greisen, Phys. Rev. Lett. 16, 748 (1966).

[26] G. T. Zatsepin and V. A. Kuzmin, Pis'ma Zh. Eksp. Teor. Fiz. 4, 114 (1966) [JETP Lett. 4, 78 (1966)].

[27] G. Sigl, Phys. Rev. D 75, 103001 (2007).

[28] G. B. Gelmini, O. Kalashev, and D. V. Semikoz, J. Cosmol. Astropart. Phys. 11 (2007) 002.

[29] M. Galaverni and G. Sigl, Phys. Rev. Lett. 100, 021102 (2008).

[30] D. Mattingly, T. Jacobson, and S. Liberati, Phys. Rev. D 67, 124012 (2003).

[31] L. Maccione and S. Liberati, J. Cosmol. Astropart. Phys. 08 (2008) 027.

[32] M. Galaverni and G. Sigl, Phys. Rev. D 78, 063003 (2008).

[33] J. R. Ellis, N. E. Mavromatos, and D. V. Nanopoulos, Gen. Relativ. Gravit. 32, 127 (2000).

[34] J. Abraham et al. (Pierre Auger Collaboration), Astropart. Phys. 29, 243 (2008).

[35] E. Armengaud, G. Sigl, T. Beau, and F. Miniati, Astropart. Phys. 28, 463 (2007).

[36] J.N. Bahcall and E. Waxman, Phys. Lett. B 556, 1 (2003).

[37] T. P. A. Collaboration, Phys. Lett. B 685, 239 (2010).

[38] J. Ellis, N.E. Mavromatos, and D. V. Nanopoulos, arXiv:1004.4167. 